\title{
Una estrategia de investigación formativa para mejorar problemas ambientales
}

\section{A strategy for training research to improve environmental problems}

\section{Uma estratégia de pesquisa formativa para melhorar os problemas ambientais}

\author{
Lorena Agudelo Obando \\ Fecha de recepción: 24/04/2018 \\ Fecha de evaluación: 01/06/2018 \\ Fecha de aceptación: 20/06/2018 \\ Disponible en línea: \\ DOI: https://doi.org/10.18359/reds.4362
}

Cómo citar este artículo:

Agudelo, L. (2018). Una estrategia de investigación formativa para mejorar problemas ambientales. Revista Educación y Desarrollo Social, 12(2), 75-84.

Licenciada en Filosofía e Historia. Especialista en Docencia Universitaria. Docente y apoyo a semilleros de investigación del Instituto Tecnológico del Putumayo, Mocoa, Colombia. Correo electrónico: lorena.agudelo@itp.edu.co 


\title{
Resumen
}

La autora sistematizó las experiencias de los estudiantes que durante nueve años en el semillero de investigación "Hinchas del medio ambiente", se familiarizaron con los fundamentos, métodos, técnicas y metodologías de la investigación para lograr un acercamiento a las comunidades en busca de mejorar ciertos problemas de carácter ambiental, como estrategia de investigación formativa en el Instituto Tecnológico del Putumayo. El artículo afirma que la actividad en el semillero puede contribuir para que los ingenieros participantes culminaran sus estudios con una formación humanística, o plus, que les abriera otros campos de actividad y fuera de gran ayuda para su quehacer profesional.

Palabras clave: encuestas; entrevistas; experiencias; investigación formativa; semillero de investigación.

\begin{abstract}
The author systematized the experiences of the students who for nine years in the research group "Fans of the environment", became familiar with the fundamentals, methods, techniques and methodologies of the research to achieve an approach to the communities in search of improving certain environmental problems, as a training research strategy at the Putumayo Technological Institute. The article states that the activity in the nursery can contribute to the participating engineers to complete their studies with a humanistic training, or plus, that will open other fields of activity and be of great help for their professional work.
\end{abstract}

Keywords: seedbed, formative research, experiences, interviews, surveys.

\section{Resumo}

Este artigo resume as experiências dos alunos na incubadora de pesquisa "Fanáticos pelo meio ambiente" que, por nove anos, familiarizaram-se com princípios, métodos, técnicas e metodologias de pesquisa voltados para a abordagem de problemas ambientais como estratégia de pesquisa formativa no Instituto Tecnológico. de Putumayo. O artigo afirma que a incubadora pode contribuir para que os engenheiros participantes concluam seus estudos com formação humanista, o que pode se tornar uma porta de entrada para outros campos de atividade e instrumental em seu trabalho profissional.

Palavras-chave: Pesquisas, entrevistas, experiências, pesquisa formativa, incubadora de pesquisa 


\section{Introducción}

En los estudios que se imparten en los institutos de educación superior se ha venido dando importancia a la experiencia y, en especial, al acercamiento a la comunidad, en busca de identificar problemas y plantear soluciones en forma conjunta, resaltando la responsabilidad social que debe caracterizar al profesional de las últimas décadas, teniendo en cuenta que representa su compromiso ante la sociedad. Así, es deseable que un profesional íntegro esté atento a los problemas que aquejan su comunidad y a buscar la forma de solucionarlos. Este fue el razonamiento que impulsó a la autora cuando decidió crear una estrategia de investigación formativa para mejorar problemas ambientales de la comunidad de Mocoa, lugar en el que se encuentra inmerso el Instituto Tecnológico del Putumayo.

Como punto de partida, el semillero "Hinchas del medio ambiente" fue el grupo por medio del cual la docente Lorena Agudelo creó una particular estrategia de investigación formativa en el Instituto Tecnológico del Putumayo, familiarizando a los estudiantes de ingeniería con los fundamentos, métodos, técnicas y metodologías de la investigación, en un intento por conseguir un acercamiento a las comunidades aledañas y mejorar algunos problemas de carácter ambiental. El primer resultado de esta estrategia de investigación fue el interés de los estudiantes por compenetrarse con los problemas ambientales circundantes y encontrar sentido a su futuro ejercicio profesional. Por supuesto, llevar a cabo el programa de investigación tuvo serios inconvenientes y tropiezos que fueron superados gracias al entusiasmo de los mismos participantes.

Al ponerse en marcha el semillero, su actividad demostró que es urgente cambiar la actitud pasiva del estudiante y transformarla en un motor de indagación circundante que identifique problemas para después buscar la forma de solucionarlos en asocio con la comunidad y los recursos que se tengan a disposición. También se vio la necesidad de resaltar el perfil de líder de los participantes, con el fin de empoderarlos para que sean agentes de los cambios urgentes que requiere el compromiso con los aspectos ambientales del planeta y concretamente de la zona del Putumayo.

Hoy se habla de estrategia para designar planes, debido a que se considera como un plan que se adelanta en una serie de pasos cuyo fin es lograr un objetivo, beneficioso en la mayoría de los casos. Por lo tanto, una estrategia es producto de la inteligencia o raciocinio que analiza una situación y busca los pasos metódicos para mejorarla o sustituirla. Ese fue el tipo de labor que adelantó la docente para poner en marcha una estrategia de investigación formativa, con la cual los participantes en el semillero "Hinchas del medio ambiente" se sintieran interesados en conocer los problemas de su comunidad e intentaran solucionarlos. Animó a sus discípulos para adentrarse en un programa diferente a lo conocido hasta entonces, contó con su compromiso más 
que con su aceptación y los involucró en un plan de reconocimiento ambiental que interesó a todos por ser claro, motivante, coherente, compatible con el entorno, adecuado a los recursos disponibles, con riesgos controlados, adecuado al temperamento juvenil y, en especial, viable para el Instituto Tecnológico del Putumayo.

En este sentido, y a propósito de los nuevos modelos transformadores que se deben dar desde la educación, Wild (2015) presta especial atención a lo que las diferentes corrientes pedagógicas defienden en sus postulados y, en contexto de los cambios sociales, considera que "la capacidad de creación, de iniciativa propia y flexibilidad en nuevas situaciones será más importante que saber cosas".

Cada día son más los docentes que buscan transformar sus prácticas en el aula, y algunas de ellas se enfocan en dejar crecer emocional e intelectualmente al estudiante, pero para dejar ser al niño primero se debe dejar ser al docente, y sin el ánimo de hacer juicios frente a lo que las diferentes políticas educativas y modelos pedagógicos defienden, sí es necesario que se articulen dos cosas: una de ellas hace referencia a los lineamientos generales de las políticas educativas y la otra a la práctica docente. Es decir, en términos coloquiales, que no se impongan políticas a espaldas de la realidad en el aula; no se puede seguir pensando en que las leyes las hagan personas que no han "dictado clase"

78 y que pretendan coaccionar la autonomía en la relación docente-estudiante, que es un elemento importante para que se den los aprendizajes.
Para Guillén (2017), "es muy importante conocer las bases neurobiológicas del aprendizaje para no cometer errores en las reformas de nuestro sistema educativo". Igualmente, el cerebro intelectual y el emocional están conectados, y a partir de los descubrimientos que ha hecho la neuroeducación se elaboró la propuesta denominada "aprender con todo nuestro potencial". No solo hay una reevaluación de cómo se aprende, sino también una necesidad de entender que el niño es más que cerebro y que lo emocional incide en el conocimiento. Estos procesos tienen marcado el hecho de que, si bien todos tenemos la capacidad de aprender, no todos llegamos al conocimiento de la misma manera, y es necesario entender la diferencia. No en sentido excluyente ni con el ánimo de rotular, pero en palabras de Guillén: ¿necesita el cerebro emocionarse para aprender? Cuarenta años atrás los psicoanalistas Reich y Schmid (1973) habían advertido en sus escritos que "El hombre tiene derecho a conocer, aprender, preguntar y cometer errores, se deben investigar las emociones humanas, por supuesto, de forma segura, y la palabra 'libertad' nunca debe ser más que un eslogan político vacío".

En la experiencia del semillero los jóvenes enfrentaron la tarea de pensarse fuera del aula así como el reto de trascender los conocimientos que hasta el momento sus docentes habían depositado en su cerebro, de aprender de la realidad, de conjugar su oportunidad de tener acceso a la educación con las 
necesidades de sus comunidades $3 / 4 q u e$ eran poblaciones víctimas del conflicto armado, olvidadas por el Estado y con explicaciones colonialistas que hablan del pobre indígena colonizado, sin oportunidades y despojado de su arraigo. En este contexto, el proceso de formación en investigación recuerda lo que en su tiempo afirmó Reimer (1973): "la única forma de corregir esto es liberando la educación de las escuelas, de forma que la gente pueda aprender de verdad sobre la sociedad donde vive" (p. 145).

Y en el tema ambiental, el indígena se siente por encima de lo que él llama "colono", porque en su pueblo, en su comunidad, en su vereda, la naturaleza tiene un papel importante y su cosmovisión está ligada a ello. Este elemento fue aprovechado por los integrantes del grupo de investigación, no solo para entender la relación que ellos mismos tenían con el territorio sino también como una forma de hablar desde aquello que para la comunidad era importante, ayudar a dar soluciones, teniendo en cuenta lo que les ofrecía el medio o la población impactada. Ese fue el valor agregado de la experiencia relatada. Este trabajo es valioso porque es un llamado a la innovación de los recursos que poseen los docentes para interesar a sus estudiantes e involucrarlos en los problemas de su comunidad.

\section{Revisión literaria}

Si bien es cierto que la metodología en la investigación y en la educación es un tema que ha tomado relevancia en los últimos treinta años, no se puede decir que este proyecto haya tenido similares llevados a textos formales. En cierto modo, este proyecto es novedoso y representa una idea imitable, porque fue diseñado para cubrir la necesidad de despertar el interés por el tema medioambiental en la región suroriental de Colombia, y fue avanzando y consolidándose con cada nueva actividad sin tener una estructura administrativa determinada.

Sin embargo, es conveniente resaltar que la docencia es un campo experimental dentro del cual el profesor puede aprovechar su inventiva y capacidad para despertar interés en temáticas locales que, muchas veces, como en este caso, llegan a superar las expectativas iniciales. En la actualidad, la docencia, como otras actividades, requiere una gran dosis de administración para lograr los resultados esperados en una capacitación, es decir, generando beneficios para los estudiantes y para el capacitador, logrando satisfacción entre todos sus participantes.

\section{Metodología}

La práctica de la docencia en Colombia enfrenta numerosos problemas que la aquejan desde diversos ángulos: la apatía de los estudiantes, la falta de investigación, los escasos recursos pedagógicos y el desinterés de los centros de enseñanza, entre otros.

La metodología de investigación empleada para elaborar el presente trabajo 
fue de tipo histórico y analítico sobre hechos cumplidos, y a partir de ello se proyectaron acciones tendientes a trabajar de manera conjunta entre el nuevo profesional y su comunidad en problemas relacionados con el medio ambiente. Por este motivo fue necesario el desplazamiento a lugares del departamento del Putumayo (como Villagarzón, Mocoa, Puerto Asís, Puerto Caicedo, La Hormiga, Orito) y lugares vecinos como Popayán, Cali, La Cruz (Nariño) y Suaza (Huila), en donde algunos jóvenes que se formaron en el Instituto Tecnológico del Putumayo compartieron sus conocimientos como ingenieros ambientales y, en especial, se apersonaron de la misión de fortalecer la educación ambiental en los jóvenes del sector rural y ahora trabajan para identificar la problemática y despertar el interés hacia el aprovechamiento y la protección del medio ambiente.

La sistematización del proyecto reconstruye un proceso de formación, en el cual docentes y estudiantes lograron resignificar sus vivencias, experiencias e intencionalidades. Cuenta la historia del semillero de investigación, la caracterización de los actores, las regiones en donde hoy, ya como ingenieros, están ofreciendo sus conocimientos, el tiempo en su proceso de formación y los proyectos en los cuales participaron, así como los valores que, después de nueve años de trabajo en esta propuesta de semillero, sus integran80 tes consideran que son importantes para el Instituto y para su vida profesional. Y, finalmente, presenta la mirada desde la institución sobre la relación que existe entre sus políticas de investigación y la experiencia del semillero como medio de formación investigativa.

Al final, se acerca este proceso de sistematización a lo que significa la investigación formativa para un centro de estudios superiores, las comunidades y el estudiante, y a cómo desde el aula de clase los docentes pueden desarrollar diferentes proyectos que se pueden convertir en ambientes para el aprendizaje, en los que los jóvenes convierten la información en conocimiento. Bruner (1988) menciona el conocimiento por descubrimiento, en el cual el aprendiente adquiere los conocimientos por sí mismo y, en este concepto, aparece un estudiante activo en su proceso formador.

\section{Resultados}

Luego de observar lo que significó esta experiencia para los actores del semillero, los valores que desarrollaron, los lugares en donde se encuentran trabajando y los diferentes proyectos y actividades que desempeñaron, cabe resaltar que este proceso estuvo ligado y aportó a la política de investigación del Centro de Investigación y Extensión Científica y Tecnológica (Ciecyt , 2015), el cual concibe la investigación como un "proceso continuo, sistemático, serio y riguroso en conceptos y metodologías para generar y construir el saber científico, tecnológico, humanístico y artístico"

Esta experiencia de enseñanza-aprendizaje se enmarca dentro de las llamadas 
pedagogías alternativas, en las cuales el docente enfatiza su labor en la manera en que se aprende. Es una forma de orientar de un modo más reflexivo, dialógico y humano, en la cual los estudiantes se preocupan por aprender en un ambiente colaborativo y solidario. Se trabajó por proyectos, y con estos se fortalecieron lazos afectivos entre los actores y la comunidad intervenida. Cada proyecto estuvo pensado y desarrollado dentro de una praxis de interacción orientada a reconocer las problemáticas ambientales, la toma de conciencia y las alternativas para solucionarlas.

La investigación formativa es un proceso en el cual docentes y estudiantes logran desarrollar una cultura investigativa sobre un tema puntual. En el caso específico del semillero "Hinchas del medio ambiente", adicional a los métodos y técnicas propias de la investigación, su proceso formador fue mediado por el trabajo comunitario en el cual los jóvenes aplicaron las herramientas adquiridas en el aula para comprender los problemas sociales y ambientales que aquejan a las comunidades, y, en un trabajo participativo, desarrollaron estrategias para su solución. La forma como se llegó al conocimiento sigue siendo una pregunta que obtiene respuestas en su tiempo, en las necesidades sociales, y se encuentra asociada a las características generacionales, sobre todo en territorios como el departamento del Putumayo, en el que se conjugan culturas indígenas que aún conservan tradiciones que dan fe del valor histórico de sus municipios y de la marca que ha dejado la violencia en esta parte de Colombia.

El escenario en el que se desarrolló la práctica docente fue el municipio de Mocoa, específicamente en el Instituto Tecnológico del Putumayo, lugar al que llegan todos los jóvenes del departamento del con el sueño de convertirse en "profesionales del mañana". Es un departamento biodiverso, el llamado pulmón del mundo, que ofrece grandes riquezas naturales y despierta en su gente la necesidad de salvaguardarlo.

Fueron varios los aspectos que enriquecieron esta experiencia, y uno de ellos fue el trabajo con la comunidad rural, conformada por indígenas y colonos con un componente muy especial: el conocimiento empírico, el cual se conjugó con el conocimiento científico, y dentro de este dualismo los jóvenes fortalecieron su vocación profesional y se formaron en investigación.

Los jóvenes, hoy ingenieros ambientales, cuentan su experiencia en este espacio formativo y resaltan que la curiosidad y el deseo de innovar y aprender los llevó a integrar el semillero y, que aún sin saber en qué consistía la investigación formativa, porque en el contexto del Instituto Tecnológico del Putumayo este tema era nuevo, ellos empezaron a desarrollar una serie de actividades que los fueron comprometiendo. Poco a poco, ese deseo de aprender fue permeado por el amor, la amistad y un sinnúmero de valores que fortalecieron el grupo y 
despertaron su sentido de pertenencia con la institución.

Dentro de los proyectos desarrollados estuvo el de "Hábitos ambientales de los niños del sector rural del municipio de Mocoa". Por medio de este ejercicio de investigación se impactaron siete veredas del sector rural que pertenecen al Centro Educativo Rural Caliyaco. El eje articulador para el desarrollo de hábitos fue la escuela, y en cuanto a la población se trabajó con los niños, docentes y padres de familia.

Se articularon dos elementos importantes: la práctica docente, desde el espacio académico sociología rural, y la formación en investigación de los estudiantes del Instituto Tecnológico del Putumayo, quienes empezaron a hacer presencia en las escuelas como orientadores ambientales y con el tiempo se ganaron el cariño de los niños.

En cuanto a la población, cabe resaltar que se trabajó con 450 niños en etapa escolar, integrados en la metodología de la escuela activa. Con ellos se desarrolló una serie de actividades orientadas al cuidado de su entorno y los recursos naturales. Con juegos, canciones, títeres y demás lúdicas, ellos fueron tomando consciencia de la necesidad de establecer una nueva relación entre hombre y naturaleza.

La experiencia también permitió el aprovechamiento de los saberes indígenas; su 82 conocimiento empírico y su cosmovisión frente al territorio fueron fundamentales para entender las problemáticas ambientales y construir soluciones para su transformación.

En un trabajo colaborativo entre la comunidad y los estudiantes de saneamiento ambiental se fueron implementando acciones para mejorar problemáticas como la deforestación, el manejo y aprovechamiento de los residuos sólidos, la protección de las fuentes hídricas, el uso eficiente del recurso hídrico, entre otros. Este proceso se enmarcó en una metodología de trabajo diseñada por la autora, en la que se establecieron tres momentos para la investigación, el trabajo comunitario y la toma de consciencia: explorando e identificando; reflexionando y preguntando; y discutiendo y solucionando. La intención era que se partiera de un diagnóstico participativo en el que los niños y la comunidad reconocieran las problemáticas ambientales de su entorno y concertaran soluciones que estuvieran acordes con los recursos que tenían a su disposición.

\section{Conclusiones}

El objetivo del estudiante es aprender, y los docentes deben orientar sus esfuerzos en proporcionarle experiencias y recursos para ayudarle en su proceso de empoderamiento del conocimiento. Este proceso está mediado por la motivación, y los semilleros son espacios en los que el joven universitario desarrolla habilidades y acepta responsabilidades sin las cuales la labor del docente sería en vano; es decir, solo el aprendiente es responsable de su progreso. Desde esta 
mirada, se habla de aprender a aprender $\mathrm{y}$ de aprender a investigar investigando. Sin embargo, cuando se reflexiona sobre los estándares de calidad y la política de investigación nacional, se observa que allí se habla abiertamente de investigación científica y tecnológica, y de esta manera se califican las universidades. Es de anotar que en los procesos formativos que se viven al interior de los semilleros no siempre los productos cumplen con estos dos requisitos, pero no por ello deja de ser importante el hecho de que los estudiantes lleguen al aprendizaje relacionando los contenidos del aula con su experiencia personal, los conocimientos adquiridos en un espacio académico con los de otros espacios o la simple actividad de resignificar las vivencias que hayan tenido, como es el caso del semillero "Hinchas del medio ambiente".

En el proceso de recolección de información para el proyecto de sistematización de las experiencias del semillero de investigación "Hinchas del medio ambiente", que hizo posible el documento Del aula a la comunidad, los participantes (desde 2009 a 2016) fueron entrevistados y como producto de estas entrevistas se evidenció que la gran mayoría de ellos hablaba de un "no haber vivido la experiencia como se debía". Sin embargo, ese "debía" viene dado por el sentimiento o la toma de conciencia de que hoy, ya como ingenieros, valoran todas las herramientas y aprendizajes que el Instituto les brindó desde el semillero y que, muy seguramente, por su juventud no le dieron ese significado en su momento. Con todo, en los procesos de formación, una respuesta como esta es valiosa porque permite entender que el estudiante en último semestre no se puede considerar como un producto terminado, porque es después de la graduación cuando todas las piezas se van encajando en su mente.

En el proceso de elaboración del material visual, producto de la sistematización, los participantes del semillero, a medida que van contado sus experiencias, dejan ver que por medio del trabajo realizado fueron creciendo como personas y que el contacto con la comunidad los ha sensibilizado frente a las necesidades del "otro" y el entendimiento de que son los profesionales de diferentes disciplinas los llamados a participar en los procesos de gestión de las acciones que mejoran la calidad de vida, en este caso, del sector rural del municipio de Mocoa. Al respecto, y teniendo en cuenta la dinámica en donde se desarrolló esta experiencia, Freire (1969) manifiesta que "es preciso que el hombre nuevo sea capaz de comprender las consecuencias globales de los comportamientos individuales, de concebir las prioridades y de asumir las solidaridades que componen el destino de la especie" (p. 32). Este comprender los comportamientos individuales le da sentido a los proyectos que han impactado a las diferentes comunidades y ratifica la necesidad de que la educación de hoy debe llevar a un conocimiento social responsable. 


\section{Referencias}

Bruner,J. (1988). Realidadmental y mundosposibles: los actos de la imaginación que dan sentido a la experiencia. Madrid: Gedisa.

Centro de Investigación y Extensión Científica y Tecnológica (Ciecyt). (2015). «Antecedentes. Instituto Tecnológico del Putumayo». Recuperado de http:// www.itp.edu.co/web2016/index.php/ investigacion\#antecedentes

Freire, P. (1969). Pedagogia da autonomia. São Paulo: Paz e Terra.

Guillén,J. (2017). Neuroeducación en el aula. De lateoría ala práctica. Madrid: CreateSpace

Reich, W. y Shmid, V. (1973). Psicoanálisis y educación. Barcelona: Anagrama.

Reimer, E. (1973). La escuela ha muerto. Barcelona: Barral.

Wild, R. (2015). Educar para ser. Vivencias de una escuela activa. Madrid: Herder. 